\title{
THE MAXIMAL IDEAL SPACE OF ALGEBRAS OF BOUNDED ANALYTIC FUNCTIONS ON INFINITELY CONNECTED DOMAINS( $\left.{ }^{1}\right)$
}

\author{
BY \\ MICHAEL FREDERICK BEHRENS
}

1. Introduction. Let $D$ be a domain in the complex plane, and let $H^{\infty}(D)$ be the algebra of bounded analytic functions on $D$. When equipped with the norm of uniform convergence, $H^{\infty}(D)$ becomes a uniform algebra, whose maximal ideal space will be denoted by $\mathscr{M}(D)$. The domain $D$ can be identified with an open subset of $\mathscr{M}(D)$, by identifying a point $\lambda \in D$ with the homomorphism "evaluation at $\lambda$." Each function in $H^{\infty}(D)$ has a natural continuous extension on $\mathscr{M}(D)$, which is given by its Gelfand transform.

There are two questions associated with $\mathscr{M}(D)$ which arise naturally. The first is the corona question, which asks whether $D$ is dense in $\mathscr{M}(D)$. Carleson [2] proved that the open unit disk $\Delta$ is dense in $\mathscr{M}(\Delta)$. Stout [9] and others extended Carleson's theorem to finitely connected planar domains, and, more generally, to finite open Riemann surfaces. We will present a technique which gives some information on this count for infinitely connected domains, and which shows that the corona conjecture is true for a certain class of infinitely connected domains. And we will show that if the corona conjecture fails for some domain, then it already fails for domains of particularly simple types, for instance, for a domain obtained from the open unit disk by excising a sequence of disjoint closed subdisks which converge to a prescribed point. The technique involves expressing $H^{\infty}(D)$ as essentially a countable direct sum of the algebra $H^{\infty}(\Delta)$, and invoking Carleson's result.

The second question involves determining to what extent an analytic structure can be introduced into subsets of $\mathscr{M}(D)$. Define an analytic disk in $\mathscr{M}(D)$ to be the image of $\Delta$ under any one-to-one map $T$ of $\Delta$ into $\mathscr{M}(D)$ which has the property that $\hat{f} \circ T$ is analytic for each $f \in H^{\infty}(D)$. Define an analytic set in $\mathscr{M}(D)$ to be any connected set which is a union of analytic disks. Then $D$ is an analytic subset of $\mathscr{M}(D)$, and it is natural to ask what the other analytic subsets of $\mathscr{M}(D)$ are.

To approach this problem, the notion of a Gleason part is decisive. Two homomorphisms $\varphi, \psi \in \mathscr{M}(D)$ are said to be in the same Gleason part if

$$
\sup \left\{|\varphi(f)-\psi(f)| \mid f \in H^{\infty}(D),\|f\| \leqq 1\right\}<2 .
$$

Received by the editors April 17, 1970.

AMS 1970 subject classifications. Primary 30A98, 46J15, 46J20.

Key words and phrases. Algebras of bounded analytic functions, corona theorem, Gleason parts, infinitely connected domains.

( $\left.{ }^{1}\right)$ Research partially supported by NSF Grant GP-7710. 
By Schwarz's lemma, each analytic subset of $\mathscr{M}(D)$ is contained in a single Gleason part. The basic problem of analytic structure in $\mathscr{M}(D)$ might be phrased as follows: Are the Gleason parts of $\mathscr{M}(D)$ all analytic subsets of $\mathscr{M}(D)$; and, if so, in what way does the analytic structure of each Gleason part reflect the analytic structure of $D$ ?

Now the first information on the parts of $\mathscr{M}(\Delta)$ is contained in the paper of I. J. Schark [8], where it is shown that $\mathscr{M}(\Delta)$ contains analytic disks distinct from $\Delta$. In [5], Hoffman showed that each Gleason part of $\mathscr{M}(\Delta)$ is either a single point or an analytic disk. Later, in [7] Hoffman gave a description of exactly how the analytic disks in $\mathscr{M}(\Delta)$ arise. He also indicated that his description carried over to $\mathscr{M}(D)$ whenever $D$ is a finite open Riemann surface. One of our principal objects is to obtain information on this point for a class of infinitely connected domains. Up to now, these are the only infinitely connected domains for which a reasonably complete description of $\mathscr{M}(D)$ is known.

The domains which we will consider in this paper are obtained as follows. Let $V$ be a domain in the complex plane and let $\left\{\Delta_{n}\right\}$ be a sequence of closed disks contained in $V$ with centers $\alpha_{n}$ which cluster on $\partial V$. We say that the $\Delta_{n}$ are hyperbolically-rare if there are disjoint closed disks $D_{n}$ with centers $\alpha_{n}$ such that $\Delta_{n} \subset D_{n} \subset V$, and such that

$$
\sum \frac{\operatorname{rad} \Delta_{n}}{\operatorname{rad} D_{n}}<\infty
$$

Note that by shrinking slightly the radii of the $D_{n}$ we may further assume that

$$
\lim _{n \rightarrow \infty} \frac{\operatorname{rad} D_{n}}{d\left(\alpha_{n}, \partial V\right)}=0 .
$$

We will show that if the corona conjecture holds for $V$ and $U$ is obtained from $V$ by deleting a hyperbolically-rare sequence of disks then the corona conjecture holds for $U$. Further, in this case, each Gleason part of $\mathscr{M}(U)$ is a single point, an analytic disk, or is obtained by identifying with each point of a Gleason part of $\mathscr{M}(V)$ the origins of a number of distinct analytic disks. In particular, if each Gleason part of $\mathscr{M}(V)$ is an analytic subset, then each Gleason part of $M(U)$ is an analytic subset.

Perhaps the simplest infinitely connected domains are obtained by deleting from the open unit disk a sequence of disjoint closed disks with centers on the positive axis which accumulate only at the origin. These domains, called $L$-domains, were studied by Zalcman in [10]. Let $D$ be such a domain obtained by deleting the disks $\Delta_{n}$ with centers $\alpha_{n}$ and radii $r_{n}$. Zalcman showed that if $\sum r_{n} / \alpha_{n}<\infty$, then

$$
\Phi_{0}(f)=\frac{1}{2 \pi i} \int_{\partial D} \frac{f(\xi)}{\xi} d \xi
$$

defines a homomorphism of $H^{\infty}(D)$, and that this homomorphism is in the same Gleason part as $D$. In the announcement [1] we consider the case when $\sum r_{n} / \alpha_{n}<\infty$ 
and $\alpha_{n+1} / \alpha_{n}<c<1$ for some constant $c$ and all $n$. In this case the $\Delta_{n}$ are hyperbolically-rare in $\Delta \mid\{0\}$. We show that the Gleason part containing $D$ is equal to the disjoint union of $D$ and a set homeomorphic to $\hat{\beta}(N) \times \Delta$ with $\hat{\beta}(N) \times\{0\}$ identified to $\Phi_{0}$. Further each $f \in H^{\infty}(D)$ is analytic on each "slice" of $\hat{\beta}(N) \times \Delta$. All other Gleason parts are single points or analytic disks. Here $\beta(N)$ is the Stone-Čech compactification of the integers $N$, and $\hat{\beta}(N)=\beta(N) \backslash N$.

Our analysis of $H^{\infty}(U)$, when $U=V \backslash \cup \Delta_{n}$ with $\left\{\Delta_{n}\right\}$ hyperbolically-rare in $V$, will follow the following outline. In $\S 2$ we write each $f \in H^{\infty}(U)$ as a sum of Cauchy integrals around the holes $\Delta_{n}$ and describe the relationship between the boundary behavior of $f$ and the behavior of this set of Cauchy integrals. In $\S 3$, we show that there is a natural embedding $\Phi$ of $\mathscr{M}(V) \mid V$ into $\mathscr{M}(U)$ with the property that for each $\psi \in \mathscr{M}(V) \backslash V, \Phi(\psi)$ is an extension of $\psi$ to $H^{\infty}(U)$. In $\S 4$ we study the algebra $H^{\infty}(\Delta \times N)$ of bounded functions on $\Delta \times N$ which are analytic on each "slice" $\Delta \times\{n\}$. Let $A_{z_{0}}(U)$ be the fiber algebra of restrictions of functions in $H^{\infty}(U)$ to the set of homomorphisms in $\mathscr{M}(U)$ which take the value $z_{0}$ on the coordinate function $z$. In $\S 5$ we show how to represent $A_{z_{0}}(U)$ as a subalgebra of $H^{\infty}(\Delta \times N) \oplus A_{z_{0}}(V)$, modulo a closed ideal. In $\S 6$ we use this representation to prove the corona theorems for $H^{\infty}(U)$ and to describe the Gleason parts of $H^{\infty}(U)$. In $\S 7$ we consider the special case $V=\Delta$. Finally in $\S 8$ we consider to what extent our methods work when sets more general than closed disks are removed from $\Delta \mid\{0\}$.

2. Decomposition of $H^{\infty}(U)$. From now on we assume that $V$ is a complex domain for which the corona theorem holds, and that $U$ is the domain obtained from $V$ by deleting the hyperbolically-rare sequence of disks $\left\{\Delta_{n}\right\}$ contained in $V$. Our analysis of $H^{\infty}(U)$ will be based on the following lemma.

LEMMA 2.1. If $\varepsilon>0$ and $M>0$ are given, there exists an integer $N$ such that: If $f_{n} \in H^{\infty}\left(\Delta_{n}^{c}\right), f_{n}(\infty)=0$, and $\left\|f_{n}\right\| \leqq M$, then

$$
\begin{array}{ll}
\sum_{m \geqq N}\left|f_{m}(z)\right|<\varepsilon, & z \notin \bigcup_{n \geqq N} D_{n}, \\
\sum_{m \geqq N ; m \neq n}\left|f_{m}(z)\right|<\varepsilon, & z \in D_{n} .
\end{array}
$$

Proof. Here $\Delta_{n}^{c}$ is the complement of $\Delta_{n}$ on the Riemann sphere. Define $L_{n}(z)$ $=\left(\operatorname{rad} \Delta_{n}\right) /\left(z-\alpha_{n}\right)$. We have $\left|L_{n}(z)\right| \leqq\left(\operatorname{rad} \Delta_{n}\right) /\left(\operatorname{rad} D_{n}\right)$ for $z \notin D_{n}$. If $f_{n} \in H^{\infty}\left(\Delta_{n}^{c}\right)$ with $\left\|f_{n}\right\| \leqq M$ and $f_{n}(\infty)=0$, Schwarz's lemma gives that $\left|f_{n}\right| \leqq M\left|L_{n}\right|$ in $\Delta_{n}^{c}$. Hence if we choose $N$ such that $\sum\left(\operatorname{rad} \Delta_{n}\right) /\left(\operatorname{rad} D_{n}\right)<\varepsilon / M$ then for $z \notin \bigcup_{n \geqq N} D_{n}$ we have

$$
\sum_{n \geqq N}\left|f_{n}(z)\right| \leqq \sum_{n \geqq N} M\left|L_{n}(z)\right| \leqq M \sum_{n \geqq N} \frac{\operatorname{rad} \Delta_{n}}{\operatorname{rad} D_{n}}<\varepsilon,
$$

proving the first statement of the lemma. The second is proved similarly.

For $f \in H^{\infty}(U)$ and $n \geqq 1$ we define

$$
P_{n}(f)(z)=\frac{1}{2 \pi i} \int_{\partial \Delta_{n}} \frac{f(\xi) d \xi}{z-\xi}, \quad z \in \Delta_{n}^{c}
$$


$P_{n}$ is a projection of $H^{\infty}(U)$ onto the functions in $H^{\infty}\left(\Delta_{n}^{c}\right)$ which vanish at $\infty$. If $f \in H^{\infty}(U)$ extends analytically to $U \cup \Delta_{n}$ then $P_{n}(f) \equiv 0$. In particular, we have

$$
P_{j} P_{k}=0 \text { if } j \neq k \text {. }
$$

Let $\delta$ be chosen such that for every $n$ the disk of radius $(1+\delta) \operatorname{rad} \Delta_{n}$ and center $\alpha_{n}$ is contained in $U \cup \Delta_{n}$. We have in this disk minus $\Delta_{n}$, for each $f \in H^{\infty}(U)$,

$$
f(z)=\frac{1}{2 \pi i} \int_{\left|\xi-\alpha_{n}\right|=(1+\delta) \operatorname{rad} \Delta_{n}} \frac{f(\xi) d \xi}{z-\xi}-P_{n} f(z) .
$$

Hence on $\partial \Delta_{n}$ we have

$$
\begin{aligned}
\left|P_{n} f(z)\right| & \leqq|f(z)|+\frac{1}{2 \pi i} \int_{\left|\xi-\alpha_{n}\right|=(1+\delta) \operatorname{rad} \Delta_{n}} \frac{|f(\xi)||d \xi|}{|\xi-z|} \\
& \leqq(1+1 / \delta)\|f\| .
\end{aligned}
$$

We have shown that $\sup _{n}\left\|P_{n}\right\|<\infty$.

We can now apply Lemma 2.1 to the sequence of functions $\left\{P_{n} f\right\}$. By that lemma $\sum_{n=1}^{\infty} P_{n} f$ will converge uniformly on compact subsets of $U$ to an element of $H^{\infty}(U)$. Then $f-\sum_{n=1}^{\infty} P_{n} f$ will define an analytic function on $U$ which extends analytically to $V$. Let $P_{0} f$ be the analytic extension of $f-\sum_{n=1}^{\infty} P_{n} f$ to $V . P_{0}$ is then a projection of $H^{\infty}(U)$ on $H^{\infty}(V)$ which is orthogonal to $P_{j}$ when $j \geqq 1$.

The following lemmas describe the boundary behavior of the $P_{n} f$.

LEMMA 2.2. Let $z_{0} \in \partial V$. Given $\varepsilon>0$ there is a $\delta>0$ such that

$$
z \in U \backslash \bigcup D_{n} \text { and }\left|z-z_{0}\right|<\delta \Rightarrow\left|f(z)-\left(P_{0} f\right)(z)-\sum_{n=1}^{\infty}\left(P_{n} f\right)\left(z_{0}\right)\right|<\varepsilon \text {. }
$$

Proof. By Lemma $2.1 \sum_{n=1}^{\infty} P_{n} f$ converges uniformly on $\left(\bigcup D_{n}\right)^{c}$ and defines a continuous function there. The lemma follows.

We have already noted that in the definition of hyperbolically-rare we may assume that

$$
\lim _{n \rightarrow \infty} \frac{\operatorname{rad} D_{n}}{d\left(\alpha_{n}, \partial V\right)}=0 .
$$

By Schwarz's lemma we have that

$$
\sup \left\{\left|f(z)-f\left(\alpha_{n}\right)\right|: z \in D_{n}\right\} \leqq \frac{2 \operatorname{rad} D_{n}}{d\left(\alpha_{n}, \partial V\right)}\|f\|
$$

for each $f \in H^{\infty}(V)$. If we define the variation of $f$ on $D_{n}$ to be $\operatorname{Var}_{D_{n}}(f)$ $=\sup \left\{|f(z)-f(w)|: z, w \in D_{n}\right\}$ we thus have, for each $f \in H^{\infty}(V)$,

$$
\lim _{n \rightarrow \infty} \operatorname{Var}_{D_{n}}(f)=0 .
$$

Lemma 2.2 now gives the following lemma on cluster sets. 
LEMMA 2.3. If $z_{0} \in \partial V$ and $f \in H^{\infty}(U)$, then

$$
\mathrm{Cl}\left(P_{0} f, z_{0}, V\right)+\sum_{n=1}^{\infty}\left(P_{n} f\right)\left(z_{0}\right)=\mathrm{Cl}\left(f, z_{0}, V \backslash \bigcup_{n=1}^{\infty} D_{n}\right) .
$$

Proof. Here $\mathrm{Cl}\left(f, z_{0}, A\right)=\left\{\lambda \mid\right.$ there is a sequence $\left\{z_{n}\right\} \subset A$ with $\lim z_{n}=z_{0}$ for which $\lim f\left(z_{n}\right)=\lambda$. By Lemma 2.2 we have the above equality with $V$ replaced by $V \backslash \cup D_{n}$ on the left. As $\operatorname{Var}_{D_{n}}\left(P_{0} f\right) \rightarrow 0$ as $n \rightarrow \infty$, we can replace $V \backslash \cup D_{n}$ by $V$.

The following lemma is similar to Lemma 2.2 but describes the behavior of the $P_{n} f$ in $\bigcup D_{n}$.

LEMMA 2.4. Given $\varepsilon>0$ and $M>0$ there is an $N_{0}$ such that if $f \in H^{\infty}(U),\|f\| \leqq M$ and $n \geqq N_{0}$ then

$$
\left|f(z)-\left(P_{n} f\right)(z)-\sum_{m \neq n} P_{m} f\left(\alpha_{n}\right)\right|<\varepsilon
$$

for $z \in D_{n}$.

Proof. By Lemma 2.1 we can choose $N_{1}$ such that $\sum_{m \geqq N_{1}, m \neq n}\left|P_{m} f(z)\right|<\varepsilon / 2$ for $z \in D_{n}$ with $n \geqq N_{1}$. We can now choose $N_{0} \geqq N_{1}$, such that

$$
\operatorname{Var}_{D_{n}}\left(\sum_{m=0}^{N_{1}}\left(P_{m} f\right)\right)<\varepsilon / 2
$$

for $n \geqq N_{0}$. This $N_{0}$ works.

3. The distinguished homomorphisms. We will identify a function in $H^{\infty}(V)$ with its restriction to $U$ and, in this way, consider $H^{\infty}(V)$ to be a subalgebra of $H^{\infty}(U)$. There is a natural mapping $R$ of $\mathscr{M}(U)$ into $\mathscr{M}(V)$ which is defined by restricting each element of $\mathscr{M}(U)$ to $H^{\infty}(V) . R$ is, of course, continuous. For $\psi \in \mathscr{M}(U)$, we define the "fiber at $\psi$ " to be $\mathscr{M}_{\psi}(U)=R^{-1}(\psi) . \mathscr{M}_{\psi}(U)$ is the set of homomorphisms in $\mathscr{M}(U)$ which extend $\psi$ to $H^{\infty}(U)$. Our first theorem will tell us that each $\psi$ in $\mathscr{M}(V) \backslash V$ can be extended to $H^{\infty}(U)$ in a way which varies continuously with $\psi$. Identifying each element $\psi$ of $\mathscr{M}(V) \backslash V$ with the extension $\Phi(\psi)$ given by this theorem gives a canonical embedding into $\mathscr{M}(U) . \Phi(\psi)$ will be called the "distinguished" homomorphism at $\psi$. This definition is motivated by the example where $V=\Delta \mid\{0\}$ and the $\Delta_{n}$ have centers on the positive axis which converge to 0 . Such domains, called $L$-domains, were studied by Zalcman in [10]. He showed that, in this case,

$$
\Phi_{0}(f)=\frac{1}{2 \pi i} \int_{\partial U} \frac{f(\xi) d \xi}{\xi}
$$

defines a homomorphism of $H^{\infty}(U)$ which is in the fiber at the origin. He called this homomorphism the "distinguished" homomorphism at 0. Each function in 
$H^{\infty}(\Delta \mid\{0\})$ extends analytically to $\Delta$ so that we may identify $\mathscr{M}_{0}(V)$ with the point 0. Then

$$
\begin{aligned}
\Phi(0)(f) & =P_{0} f(0)+\sum_{n=1}^{\infty} P_{n} f(0) \\
& =\sum_{n=0}^{\infty} \frac{1}{2 \pi i} \int_{\partial \Delta_{n}} \frac{f(\xi) d \xi}{\xi}=\frac{1}{2 \pi i} \int_{\partial U} \frac{f(\xi) d \xi}{\xi} \\
& =\Phi_{0}(f) \quad\left(\Delta=\Delta_{0}\right),
\end{aligned}
$$

so that $\Phi(0)$ and $\Phi_{0}$ are the same homomorphisms.

THEOREM 3.1. For $\psi \in \mathscr{M}(V) \backslash \cup D_{n}$, define

$$
\Phi(\psi)(f)=\psi\left(P_{0} f\right)+\sum_{n=1}^{\infty}\left(P_{n} f\right)(\psi(z))
$$

for $f \in H^{\infty}(U)$. Then $\Phi$ is a homeomorphism of $\mathscr{M}(V) \cup \cup D_{n}$ into $\mathscr{M}(U)$ and $R(\Phi(\psi))=\psi$ for each $\psi \in \mathscr{M}(V) \backslash \bigcup D_{n}$.

Note. Here $z$ is the coordinate function on $V$.

Proof. $\Phi(\psi)$ is evidently a continuous linear functional on $H^{\infty}(U)$, for each $\psi \in \mathscr{M}(V) \backslash \bigcup D_{n}$, so that $\Phi$ is a bijection of $\mathscr{M}(V) \cup D_{n}$ into the dual of $H^{\infty}(U)$. If $\left\{\psi_{\lambda}\right\} \subset \mathscr{M}(V) \cup \bigcup D_{n}$ converges to $\psi$ then $\psi_{\lambda}(z)$ converges to $\psi(z)$. As $\left\{\psi_{\lambda}(z)\right\}$ will be contained in the closure of $V \backslash \bigcup D_{n}$ and each $\sum_{n=1}^{\infty} P_{n} f$ is continuous on this set, we have that $\sum_{n=1}^{\infty} P_{n} f\left(\psi_{\lambda}(z)\right)$ converges to $\sum_{n=1}^{\infty} P_{n} f(\psi(z))$ for each $f \in H^{\infty}(U)$. It follows that $\Phi$ is a continuous mapping of $\mathscr{M}(V) \cup D_{n}$ into the dual of $H^{\infty}(U)$ when this dual space is equipped with the weak* topology. Now for $\psi \in V \backslash \bigcup D_{n}$ we have $\Phi(\psi)=\psi$, so that $\Phi(\psi)$ is multiplicative for all $\psi$ in a dense subset of $\mathscr{M}(V) \cup D_{n}$ (dense by the corona theorem for $V$ ). Continuity now gives that each $\Phi(\psi)$ is multiplicative. That $\Phi(\psi) \in \mathscr{M}_{\psi}(U)$ is also now clear.

For a complex number $z_{0}$ define the "fiber at $z_{0}$ " in $\mathscr{M}(V)$ to be the set

$$
\mathscr{M}_{z_{0}}(V)=\left\{\psi \in \mathscr{M}(V) \mid \psi(z)=z_{0}\right\} \text {. }
$$

Define $\mathscr{M}_{z_{0}}(U)$ similarly. As we have identified $z_{0}$ and "evaluation at $z_{0}$ " for $z_{0} \in U$, we have given two definitions of the fiber $\mathscr{M}_{z_{0}}(U)$. These two definitions agree and we have for $z_{0} \in U$ that $\mathscr{M}_{z_{0}}(U)=\left\{z_{0}\right\}$.

THEOREM 3.2. Let $z_{0} \in \partial V$ with $z_{0}$ not in the closure of $\left\{\alpha_{n}\right\}$. Then each element of $\mathscr{M}_{z_{0}}(V)$ has a unique extension to $H^{\infty}(U)$ and $\Phi$ defines a homeomorphism of $\mathscr{M}_{z_{0}}(V)$ onto $\mathscr{M}_{z_{0}}(U)$.

Proof. Let $\psi \in \mathscr{M}_{z_{0}}(U)$. For each $f \in H^{\infty}(U), \sum_{n=1}^{\infty} P_{n} f-\sum_{n=1}^{\infty} P_{n} f\left(z_{0}\right)$ is analytic in a neighborhood of $z_{0}$ and vanishes at $z_{0}$. It follows that this function is divisible by $z-z_{0}$ and is hence in the kernel of $\psi$. Thus

$$
\psi(f)=\psi\left(P_{0} f\right)+\psi\left(\sum_{n=1}^{\infty} P_{n} f\right)=\psi\left(P_{0} f\right)+\sum_{n=1}^{\infty} P_{n} f\left(z_{0}\right)
$$

and $\psi$ is hence a "distinguished" homomorphism. 
We have noted that we may assume that $\operatorname{Var}_{D_{n}}(f) \rightarrow 0$ for each $f \in H^{\infty}(V)$. A simple consequence of this is the following:

LEMMA 3.3. If $\left\{z_{\lambda}\right\}$ is a net in $V$ which converges to $\psi \in \mathscr{M}(V) \mid V$ and each $z_{\lambda} \in \bigcup D_{n}$, then $\psi$ is in the closure of $\left\{\alpha_{n}\right\}$ in $\mathscr{M}(V)$.

We state this lemma so that we may prove the following extension of Theorem 3.2.

THEOREM 3.4. Each $\psi \in \mathscr{M}(V) \backslash V$ which is not in the closure of $\left\{\alpha_{n}\right\}$ in $\mathscr{M}(V)$ has a unique extension to $H^{\infty}(U)$ which is contained in the closure of $U$ in $\mathscr{M}(U)$.

Note. Since we will show that the closure of $U$, in $\mathscr{M}(U)$, is equal to $\mathscr{M}(U)$, the extended homomorphism of Theorem 3.4 is, in fact, unique.

Proof. Let $\left\{z_{\lambda}\right\} \subset U$ be a net which converges to an element of $\mathscr{M}_{\psi}(U)$. By Lemma $3.3\left\{z_{\lambda}\right\}$ is eventually in $V \backslash \cup D_{n}$, and hence, by Lemma 2.2 , converges to $\Phi(\psi)$.

This is a convenient place to state the following result which is due to Gamelin [4]. $A_{z_{0}}(U)$ is the algebra of restrictions of functions in $H^{\infty}(U)$ to $\mathscr{M}_{z_{0}}(U)$.

THEOREM 3.5. $A_{z_{0}}(U)$ is a uniformly closed algebra with maximal ideal space $\mathscr{M}_{z_{0}}(U)$. For each $f \in H^{\infty}(U), \hat{f}\left(\mathscr{M}_{z_{0}}(U)\right)=\mathrm{Cl}\left(f, z_{0}, U\right)$. In particular,

$$
\sup \left|\hat{f}\left(\mathscr{M}_{z_{0}}(U)\right)\right|=\sup \left|\mathrm{Cl}\left(f, z_{0}, U\right)\right| .
$$

Using Theorem 3.5 we can prove the following technical lemma which will be needed later. The mapping defined by $L_{n}(z)=\left(\operatorname{rad} \Delta_{n}\right) /\left(z-\alpha_{n}\right)$ maps the complement of $\Delta_{n}$ onto the open unit disk and is equal to 0 at $\infty$. Fix $z_{0} \in \partial V$. By Lemma 2.1, for each $P \subset N$,

$$
h_{P}=\sum_{n \in P} L_{n}-\sum_{n \in P} L_{n}\left(z_{0}\right)
$$

will converge uniformly on compact subsets of $U$ to a function in $H^{\infty}(U)$. Let $h=h_{N}$. Note that $h_{P}$ was so defined that $h_{P}+h_{P^{c}}=h$ and $\mathrm{Cl}\left(h_{P} h_{P^{c}}, z_{0}, U\right)=\{0\}$. By Theorem 3.5 this means that N.B. $\hat{h}_{P} \hat{h}_{P^{c}}$ vanishes on $\mathscr{M}_{z_{0}}(U)$.

LEMMA 3.6. If $\psi \in \mathscr{M}_{z_{0}}(U)$ is such that $\psi\left(h_{P}\right) \neq 0$, and $f \in H^{\infty}(V)$ is such that $\left|f\left(\alpha_{n}\right)\right|<\varepsilon$ for all $n \in P$, then $|\psi(f)| \leqq \varepsilon /|\psi(h)|$.

Proof. It is easy to see that $\mathrm{Cl}\left(h_{P}, z_{0}, V \backslash \cup D_{n}\right)=\{0\}$ and that $\sup \left|\mathrm{Cl}\left(h_{P}, z_{0}, U\right)\right|$ $\leqq 1$. It follows that sup $\left|\mathrm{Cl}\left(f h_{P}, z_{0}, U\right)\right| \leqq \varepsilon$. Hence, by Theorem $3.5,\left|\psi(f) \psi\left(h_{P}\right)\right| \leqq \varepsilon$. Now $\psi\left(h_{P}\right) \psi\left(h_{P^{c}}\right)=0$ and $\psi\left(h_{P}\right) \neq 0$ so that $\psi\left(h_{P^{c}}\right)=0$. From $h=h_{P}+h_{P^{c}}$ it now follows that $\psi(h)=\psi\left(h_{P}\right)+\psi\left(h_{P} c\right)=\psi\left(h_{P}\right)$. We thus have that $|\psi(f) \leqq \varepsilon /| \psi\left(h_{P}\right)|=\varepsilon /| \psi(h) \mid$.

N.B. Proof. Let $z_{i} \in V, z_{i} \rightarrow z_{0}$. By passing to a subsequence we may assume that $z_{i} \in \bigcup_{n \in P} D_{n}$, say, since if all $z_{i} \notin \bigcup_{n \in P} D_{n}$, then clearly $\sum_{n \in P} L_{n}\left(z_{i}\right) \rightarrow \sum_{n \in P} L_{n}\left(z_{0}\right)$ and hence $\lim h_{P}\left(z_{i}\right)=0$. Now if all $z_{i} \in \bigcup_{n \in P} D_{n}$ then Lemma 2.1 gives

$$
\sum_{n \notin P} L_{n}\left(z_{i}\right) \rightarrow \sum_{n \notin P} L_{n}\left(z_{0}\right)
$$

and hence $\lim h_{P} c\left(z_{i}\right)=0$. 
4. The algebra $H^{\infty}(\Delta \times N)$. Let $H^{\infty}(\Delta \times N)$ be the algebra of bounded functions on $\Delta \times N$ which are analytic on each "slice" $\Delta \times\{n\} . H^{\infty}(\Delta \times N)$, when endowed with the supremum norm, is a Banach algebra. We denote the maximal ideal space of this algebra of $\mathscr{M}\left(H^{\infty}(\Delta \times N)\right)$ and consider $\Delta \times N$ to be a subset of $\mathscr{M}\left(H^{\infty}(\Delta \times N)\right)$ by identifying each $(\lambda, n) \in \Delta \times N$ with the corresponding point "evaluation at $(\lambda, n)$ " in $\mathscr{M}\left(H^{\infty}(\Delta \times N)\right)$. For each point $\psi \in \mathscr{M}(\Delta)$ and each $n \in N$ define the point $(\psi, n) \in \mathscr{M}\left(H^{\infty}(\Delta \times N)\right)$ to be that homomorphism of $H^{\infty}(\Delta \times N)$ for which $(\psi, n)(f)=\psi(f(\cdot, n))$ for each $f \in H^{\infty}(\Delta \times N)$. The set $\mathscr{M}(\Delta) \times\{n\}$ defined in this way is a subset of $\mathscr{M}\left(H^{\infty}(\Delta \times N)\right)$ which is homeomorphic to $\mathscr{M}(\Delta)$. This subset is equal to the closure of $\Delta \times\{n\}$, is both open and closed, and is the maximal connected subset of $\mathscr{M}\left(H^{\infty}(\Delta \times N)\right)$ which contains $\Delta \times\{n\}$. The set $\mathscr{M}(\Delta) \times N$ thus obtained contains those homomorphisms $H^{\infty}(\Delta \times N)$ which are independent of the behavior of each $f \in H^{\infty}(\Delta \times N)$ in the limit as $n \rightarrow \infty$. Our first theorem will describe the remaining set of homomorphisms of $H^{\infty}(\Delta \times N)$ by decomposing it in a manner similar to the decomposition of $\mathscr{M}(\Delta) \times N$ into the sets $\mathscr{M}(\Delta) \times\{n\}$.

We will identify $l^{\infty}$ with the subalgebra of $H^{\infty}(\Delta \times N)$ consisting of these functions which are constant on each "slice" $\Delta \times\{n\}$. The maximal ideal space of $l^{\infty}$ is equal to $\beta(N)$, the Stone-Čech compactification of $N$. Hence we obtain a continuous mapping $S$ of $\mathscr{M}\left(H^{\infty}(\Delta \times N)\right)$ into $\beta(N)$ by restricting each homomorphism of $H^{\infty}(\Delta \times N)$ to $l^{\infty}$. For each $\omega \in \beta(N)$ let $\mathscr{D}(\omega)=S^{-1}(\omega)$, and for each $A \subset \beta(N)$ let $\mathscr{D}(A)=S^{-1}(A)$.

THEOREM 4.1. $\{\mathscr{D}(\omega): \omega \in \beta(N)\}$ is a decomposition of $\mathscr{M}\left(H^{\infty}(\Delta \times N)\right)$ into maximal connected sets. Each $\mathscr{D}(\omega)$ is a generalized peak set. For $n \in N, \mathscr{D}(n)$ $=\mathscr{M}(\Delta) \times\{n\}$.

Proof, For each $P \subset N$ define the function $I_{P} \in H^{\infty}(\Delta \times N)$ by $I_{P}(\lambda, n)=1$ if $n \in P$ and $I_{P}(\lambda, n)=0$ if $n \in P^{c}$. We consider the point $\omega \in \beta(N)$ to be both a homomorphism of $l^{\infty}$ and an ultrafilter on $N$. It is easy to see that for each $\omega \in \beta(N)$ we have

$$
\mathscr{D}(\omega)=\bigcap_{P \in \omega} I_{P}^{-1}(1) .
$$

This shows that $\mathscr{D}(\omega)$ is a generalized peak set. Now the restriction of $H^{\infty}(\Delta \times N)$ to $\mathscr{D}(\omega)$ is a Banach algebra with maximal ideal space $\mathscr{D}(\omega)$, so that, by a general theorem on Banach algebras, to show that $\mathscr{D}(\omega)$ is connected it suffices to show that for each $f \in H^{\infty}(\Delta \times N)$ the set $\hat{f}(\mathscr{D}(\omega))$ is connected. If $\mathscr{D}(\omega)$ is connected it is obviously maximal with this property.

Let $\omega \in \beta(N)$. For each $f \in H^{\infty}(\Delta \times N)$ let $f_{\omega} \in H^{\infty}(\Delta \times N)$ be defined by $f_{\omega}(\lambda, n)=f(\lambda, n)-f(0, n)+\omega(\{f(0, \cdot)\})$. We have

$$
\hat{f}(\mathscr{D}(\omega))=\hat{f}_{\omega}(\mathscr{D}(\omega))=\bigcap_{P \in \omega} \hat{f}_{\omega}\left(\hat{I}_{P}^{-1}(1)\right)=\bigcap_{P \in \omega} \mathrm{Cl}\left(f_{\omega}\left(I_{P}^{-1}(1)\right)\right) .
$$


This last set is connected since each set $\mathrm{Cl}\left(f_{\omega}\left(I_{\vec{P}}^{-1}(1)\right)\right)$ is connected and these sets are a filter base. That $\mathscr{D}(n)=\mathscr{M}(\Delta) \times\{n\}$ for $n \in N$ is obvious.

We note that $\{\mathscr{D}(\omega): \omega \in \beta(N)\}$ is not the antisymmetric decomposition of $\mathscr{M}\left(H^{\infty}(\Delta \times N)\right)$. For if $f \in H^{\infty}(\Delta \times N)$ is such that $f(\cdot, n)$ maps $\Delta$ conformally on the rectangle with vertices $(0,0),(0,1 / n),(1,0),(1,1 / n)$ then $f$ is real and nonconstant on each $\mathscr{D}(\omega)$ with $\omega \in \hat{\beta}(N)$.

The algebra $H^{\infty}(\Delta \times N)$ in many ways resembles the algebra $H^{\infty}(\Delta)$. In particular, a corona theorem holds for $H^{\infty}(\Delta \times N)$.

THEOREM 4.2. $\Delta \times N$ is dense in the maximal ideal space $\mathscr{M}\left(H^{\infty}(\Delta \times N)\right)$ of $H^{\infty}(\Delta \times N)$.

Proof. Suppose $f_{1}, \ldots, f_{n} \in H^{\infty}(\Delta \times N)$ satisfy $\left|f_{1}\right|+\cdots+\left|f_{n}\right| \geqq \delta>0$ on $\Delta \times N$. We must find $g_{1}, \ldots, g_{n} \in H^{\infty}(\Delta \times N)$ satisfying $\sum f_{i} g_{i}=1$. By Carleson's solution of the corona conjecture for the unit disk $\Delta$, there are functions $g_{1 m}, \ldots, g_{n m}$ $\in H^{\infty}(\Delta \times\{m\})$, such that $\sum_{j=1}^{n} f_{j} g_{j m}=1$ on $\Delta \times\{m\}$, and such that $\left|g_{j m}\right| \leqq M$, where $M$ depends only on $\delta$. The $g_{j m}$ then determine functions $g_{j} \in H^{\infty}(\Delta \times N)$ which do the trick.

Now $H^{\infty}(\Delta)$ can be considered a subalgebra of $C(Y)$, where $Y$ is the maximal ideal space of $L^{\infty}(\partial \Delta, d \theta)$. In fact, $H^{\infty}(\Delta)$ is a strongly logmodular algebra on $Y$, in the sense that every $u \in C_{R}(Y)$ is equal to $\log |f|$, for some $f \in H^{\infty}(\Delta)$. Regarding $H^{\infty}(\Delta \times\{m\})$ as a subalgebra of $C(Y \times\{m\})$, we see that $H^{\infty}(\Delta \times N)$ becomes a subalgebra of the Čech compactification $\beta(Y \times N)$ of $Y \times N$.

THEOREM 4.3. $H^{\infty}(\Delta \times N)$ is a strongly logmodular algebra on $\beta(Y \times N)$.

Proof. Let $u \in C_{R}(\beta(Y \times N))$, and let $u_{m}$ be the restriction of $u$ to $Y \times\{m\}$. There is $f_{m} \in H^{\infty}(\Delta \times\{m\})$ such that $\log \left|f_{m}\right|=u_{m}$, regarded as functions on $Y \times\{m\}$. The $f_{m}$ determine a function $f \in H^{\infty}(\Delta \times N)$ such that $\log |f|=u$ on $Y \times N$ and hence on $\beta(Y \times N)$. That does it.

As a consequence of Theorem 4.3 and Hoffman's paper [5], we can state the following corollary.

CoRollary 4.4. Each Gleason part of $H^{\infty}(\Delta \times N)$ is either a single point or an analytic disk.

Define a function $Z \in H^{\infty}(\Delta \times N)$ by $Z(\lambda, n)=\lambda$. Clearly $\|Z\|=1$.

THEOREM 4.5. The subset of $\mathscr{M}\left(H^{\infty}(\Delta \times N)\right)$ on which $|\hat{Z}|<1$ is homeomorphic to $\Delta \times \beta N$.

Proof Define a mapping $G: \Delta \times \beta(N) \rightarrow \mathscr{M}\left(H^{\infty}(\Delta \times N)\right)$ by $G(\lambda, \omega)(f)=\omega(f(\lambda, \cdot))$ for all $(\lambda, \omega) \in \Delta \times \beta(N)$ and $f \in H^{\infty}(\Delta \times N)$. $G$ is obviously bijective. For each $(\lambda, n) \in \Delta \times N$ we have $G(\lambda, n)=(\lambda, n)$. We claim that $G(\Delta \times \beta(N))=Z^{-1}(\Delta)$. Clearly $G(\Delta \times \beta(N)) \subset \hat{Z}^{-1}(\Delta)$. Each element of $\hat{Z}^{-1}(\lambda)$ vanishes on the ideal of functions in $H^{\infty}(\Delta \times N)$ which vanish on $\{\lambda\} \times N$ and hence defines a homomorphism 
of $H^{\infty}(\Delta \times N) \mid\{\lambda\} \times N$. By the definition of $G$, this homomorphism must be among $G(\{\lambda\} \times \beta(N))$, so that $G(\{\lambda\} \times \beta(N)) \supset \hat{Z}^{-1}(\lambda)$ and $G(\Delta \times \beta(N)) \supset \hat{Z}^{-1}(\Delta)$.

The projection on $\Delta \times \beta(N)$ defined by $(\lambda, \omega) \rightarrow \lambda$ is equal to $Z \circ G$ and the projection on $\Delta \times \beta(N)$ defined by $(\lambda, \omega) \rightarrow \omega$ is equal to $S \circ G$. Hence $G$ is an open mapping. For $0<r<1$, let $\Delta_{r}=\{z|| z \mid \leqq r\}$. Then $G\left(\Delta_{r} \times \beta(n)\right)=\hat{Z}^{-1}\left(\Delta_{r}\right)$ is compact so that $G$ is a homeomorphism on $\Delta_{r} \times \beta(N)$. That $G$ is a homeomorphism now follows from the fact that a set $\mathcal{O}$ in $\Delta \times \beta(N)$ is open if and only if for each $r$, $\mathcal{O} \cap\left(\Delta_{r} \times \beta(N)\right)$ is open in $\Delta_{r} \times \beta(N)$.

We identify each point $(\lambda, \omega)$ with its image $G(\lambda, \omega)$.

We remark that $\Delta \times\{\omega\} \subset \mathscr{D}(\omega)$, but that $\Delta \times\{\omega\}$ is not dense in $\mathscr{D}(\omega)$. In fact, let $Y \in H^{\infty}(\Delta \times N)$ be defined by $Y(\lambda, n)=\lambda^{n}$. Then $\hat{Y}$ vanishes on each $\Delta \times\{\omega\}$ with $\omega \in \beta(N) \backslash N$ but has modulus 1 everywhere on the Shilov boundary and hence, as $\mathscr{D}(\omega)$ is a generalized peak set, at some point of $\mathscr{D}(\omega)$.

Let $H_{0}^{\infty}(\Delta \times N)$ be the subalgebra of $H^{\infty}(\Delta \times N)$ consisting of those functions which are equal to 0 on the set $\{0\} \times N . H^{\infty}(\Delta \times N)$ is equal to the Banach space direct sum of $H_{0}^{\infty}(\Delta \times N)$ and $l^{\infty}$. This decomposition is given by $f=g+h$ where $g(\lambda, n)=f(\lambda, n)-f(0, n)$ and $h(\lambda, n)=f(0, n)$. Let $\mathscr{M}\left(H_{0}^{\infty}(\Delta \times N)\right)$ be the maximal ideal space of the algebra obtained by adjoining the constants to $H_{0}^{\infty}(\Delta \times N)$. We have $H_{0}^{\infty}(\Delta \times N)=Z H^{\infty}(\Delta \times N)$.

If $\psi$ is a nonzero homomorphism of $H_{0}^{\infty}(\Delta \times N)$ then $\psi(z) \neq 0$ and $\bar{\psi}(f)$ $=\psi(z f) / \psi(z)$ defines a homomorphism of $H^{\infty}(\Delta \times N)$. To check that this is a homomorphism it suffices to show that

$$
\psi(z f g) / \psi(z)=\psi(z f) \psi(z g) /(\psi(z))^{2} .
$$

This follows from the formula $\psi(z f) \psi(z g)=\psi(z) \psi(z f g)$, which holds since $z, z f, z g$, and $z f g$ are in $H_{0}^{\infty}(\Delta \times N)$. This construction shows that the extension $\bar{\psi}$ is unique.

The space $\mathscr{M}\left(H_{0}^{\infty}(\Delta \times N)\right)$ is thus obtained from $\mathscr{M}\left(H^{\infty}(\Delta \times N)\right)$ by identifying $\hat{Z}^{-1}(0)$ to a point. We note that the extension of a homomorphism of $H_{0}^{\infty}(\Delta \times N)$ to $H^{\infty}(\Delta \times N)$ is also determined in the following way. For $P \subset N$ let $Z_{P}(\lambda, n)=\lambda$ if $n \in P$ and $Z_{P}(\lambda, n)=0$ if $n \in P^{c}$.

LEMMA 4.6. Let $\psi \in \mathscr{M}\left(H^{\infty}(\Delta \times N)\right)$. If $\psi\left(Z_{P}\right) \neq 0$ and $f \in H^{\infty}(\Delta \times N)$ satisfies $|f(z, n)| \geqq \varepsilon$ for $n \in P$ then $|\psi(f)| \geqq \varepsilon$.

Proof. Suppose that $g \in H^{\infty}(\Delta \times N)$ vanishes on $\Delta \times P$. Then $g Z_{P}=0$ so that $\psi(g) \psi\left(Z_{P}\right)=0$ and hence $\psi(g)=0$. Hence $\psi$ determines a homomorphism of $H^{\infty}(\Delta \times P)$. Since $f$ is invertible on $\Delta \times P$ with inverse bounded by $1 / \varepsilon$ we must have $|\psi(f)| \geqq \varepsilon$.

5. The isomorphism theorem. Fix $z_{0} \in \partial V$ and let $\mathfrak{A}=H^{\infty}(\Delta \times N) \oplus A_{z_{0}}(V)$ be the Banach algebra of all $(u, v)$ with $u \in H^{\infty}(\Delta \times N)$ and $v \in A_{z_{0}}(V)$, with addition and multiplication defined coordinatewise, and norm equal to $\|(u, v)\|=$ $\max (\|u\|,\|v\|)$. Identify $(u, 0)$ and $u$, and $(0, v)$ and $v . A_{z_{0}}(V)$ was defined in $\S 3$ and $H^{\infty}(\Delta \times N)$ in $\S 4$. 
We define a closed ideal $\mathscr{L}$ in $\mathfrak{A}$ as follows: $(u, v) \in \mathscr{L}$ if and only if $v \equiv 0$ and for each $\varepsilon>0$ there is a $\delta>0$ such that

$$
\left|\alpha_{n}-z_{0}\right|<\delta \Rightarrow|u(z, n)|<\varepsilon \text { for all } z \in \Delta .
$$

We are going to represent $A_{z_{0}}(U)$ as a subalgebra of $\mathfrak{A} / \mathscr{L}$.

Define a linear mapping $T: H^{\infty}(U) \rightarrow \mathfrak{A}$ by $T(f)=(u, v)$ with

$$
\begin{aligned}
u(z, n) & =P_{n} f\left(L_{n}^{-1}(z)\right)+\sum_{m \neq n} P_{m} f\left(\alpha_{n}\right), \\
v & =P_{0} f \mid \mathscr{M}_{z_{0}}(V)+\sum_{m=1}^{\infty} P_{m} f\left(z_{0}\right) .
\end{aligned}
$$

Suppose that $f \in H^{\infty}(U)$ vanishes on $\mathscr{M}_{z_{0}}(U)$. Then there is a $\delta>0$ such that $z \in U$ and $\left|z-z_{0}\right|<\delta$ implies that $|f(z)|<\varepsilon$. There is a $\delta^{\prime}>0$ such that if $\left|\alpha_{n}-z_{0}\right|<\delta^{\prime}$ and $z \in \Delta$ has modulus greater than $\frac{1}{2}$, then $L_{n}^{-1}(z) \in D_{n} \mid \Delta_{n}$ and $\left|L_{n}^{-1}(z)-z_{0}\right|<\delta$. We can also choose $\delta^{\prime}$ to have the property that $\left|\alpha_{n}-z_{0}\right|<\delta^{\prime}$ implies that $n>N_{0}$ where the $N_{0}$ is from Lemma 2.4. By Lemma 2.4 and the maximum modulus principle in $\Delta$ we now have that $\left|\alpha_{n}-z_{0}\right|<\delta^{\prime}$ implies that $|u(z, n)|<2 \varepsilon$ for all $z \in \Delta$. By Lemma 2.3

$$
\mathrm{Cl}\left(P_{0} f+\sum_{n=1}^{\infty} P_{n} f\left(z_{0}\right)\right)=\{0\}
$$

and hence, by Lemma 3.4,

$$
P_{0} f \mid \mathscr{M}_{z_{0}}(V)+\sum_{m=1}^{\infty} P_{m} f\left(z_{0}\right) \equiv 0 .
$$

Hence $(u, v) \in \mathscr{L}$ so that $T$ induces a linear mapping from $A_{z_{0}}(U)$ into $\mathfrak{A} \mid \mathscr{L}$.

THEOREM 5.1. Let $\Theta$ be the mapping $\Theta: A_{z_{0}}(U) \rightarrow \mathfrak{A} \mid \mathscr{L}$ defined by $\Theta\left(f \mid \mathscr{M}_{z_{0}}(U)\right)$ $=(u, v)+\mathscr{L}$ where

$$
\begin{aligned}
u(z, n) & =P_{n} f\left(L_{n}^{-1}(z)\right)+\sum_{m \neq n} P_{m} f\left(\alpha_{n}\right), \\
v & =P_{0} f \mid \mathscr{M}_{z_{0}}(V)+\sum_{m=1}^{\infty} P_{m} f\left(z_{0}\right) .
\end{aligned}
$$

$\Theta$ is an isometric (algebra) isomorphism of $A_{z_{0}}(U)$ onto a subalgebra of $\mathfrak{X} \mid \mathscr{L}$.

Proof. $\Theta$ is defined by $\Theta\left(f \mid \mathscr{M}_{z_{0}}(U)\right)=T(f)+\mathscr{L}$ so that $\Theta$ is a well-defined linear mapping. Let $f_{0}=f \mid \mathscr{M}_{z_{0}}(U)$ and $\left\|f_{0}\right\| \leqq M$. Lemma 2.3 and Theorem 3.5 give that $\|v\| \leqq M$. If $\delta$ is chosen such that $z \in U$ and $\left|z-z_{0}\right|<\delta$ implies that $|f(z)|<M+\varepsilon$, and $\delta^{\prime}$ is chosen for this $\delta$ as before, then Lemma 2.4 gives that $|u(z, n)| \leqq M+2 \varepsilon$ for all $z \in \Delta$. This shows that $\left\|\Theta\left(f_{0}\right)\right\| \leqq\left\|f_{0}\right\|$.

Let $z_{n}$ be a sequence in $U$ for which $z_{n} \rightarrow z_{0}$ and $f\left(z_{n}\right) \rightarrow\left\|f_{0}\right\|$. If an infinite number of the $z_{n}$ are in $U \backslash \cup D_{n}$ then Lemma 2.3 shows that $\|v\|=\left\|f_{0}\right\|$ and hence that $\left\|\Theta\left(f_{0}\right)\right\| \geqq\|v\|=\left\|f_{0}\right\|$. 
If an infinite number of the $z_{n}$ are contained in $\bigcup D_{n}$, then arguing as before using Lemma 2.4 gives that $\left\|\Theta\left(f_{0}\right)\right\| \geqq\left\|f_{0}\right\|$. Thus $\Theta$ is an isometry.

Let $f_{1}, f_{2} \in H^{\infty}(U)$ and let $T\left(f_{1}\right)=\left(u_{1}, v_{1}\right), T\left(f_{2}\right)=\left(u_{2}, v_{2}\right)$ and $T\left(f_{1} f_{2}\right)=\left(u_{3}, v_{3}\right)$. Let $f_{3}=f_{1} f_{2}$. If $\delta$ is such that $\left|\alpha_{n}-z_{0}\right|<\delta$ implies that $n$ is larger than $N_{0}$ of Lemma 2.4, the Lemma 2.4 gives, for fixed $n$ with $\left|\alpha_{n}-z_{0}\right|<\delta$ and $z \in \Delta$ with modulus close to 1 ,

$$
\left|f_{i}\left(L_{n}^{-1}(z)\right)-u_{i}(z)\right|<\varepsilon, \quad i=1,2,3 .
$$

Hence for $|z|$ close to 1 ,

$$
\begin{aligned}
\left|u_{3}(z, n)-u_{1}(z, n) u_{2}(z, n)\right| \leqq & \left|f_{1} f_{2}\left(L_{n}^{-1}(z)\right)-u_{3}(z, n)\right| \\
& +\left|\left(u_{1}(z, n)-f_{1}\left(L_{n}^{-1}(z)\right)\right) f_{2}\left(L_{n}^{-1}(z)\right)\right| \\
& +\left|u_{1}(z, n)\left(u_{2}(z, n)-f_{2}\left(L_{n}^{-1}(z)\right)\right)\right| \\
\leqq & \varepsilon\left(1+\left\|f_{2}\right\|+\left\|f_{1}\right\|+\varepsilon\right) .
\end{aligned}
$$

By the maximum modulus principle we have

$$
\left|u_{3}(z, n)-u_{1}(z, n) u_{2}(z, n)\right|<\varepsilon\left(1+\left\|f_{1}\right\|+\left\|f_{2}\right\|+\varepsilon\right)
$$

for all $n$ such that $\left|\alpha_{n}-z_{0}\right|<\delta$ and all $z \in \Delta$.

By Lemma 2.2 we will have that

$$
\left|P_{0}\left(f_{1} f_{2}\right)(z)+\sum_{n=1}^{\infty} P_{n}\left(f_{1} f_{2}\right)\left(z_{0}\right)-\left(P_{0} f_{1}(z)+\sum_{n=1}^{\infty} P_{n} f_{1}(z)\right)\left(P_{0} f_{2}(z)+\sum_{n=1}^{\infty} P_{n} f_{2}\left(z_{0}\right)\right)\right|
$$

goes to 0 uniformly as $z \rightarrow z_{0}$ for all $z \in U \backslash \cup D_{n}$. This limit then goes to 0 as $z \rightarrow z_{0}$ for all $z \in V$ so that, by Theorem 3.5, this function vanishes on $\mathscr{M}_{z_{0}}(V)$, and hence $v_{3}=v_{1} v_{2}$. We are done.

THEOREM 5.2. Each homomorphism of $\Theta\left(A_{z_{0}}(U)\right)$ is the restriction of a homomorphism of $\mathfrak{A} \mid \mathscr{L}$.

Proof. Let $m_{0} \in \mathscr{M}_{z_{0}}(U)$. We first consider the case when $m_{0}$ is a distinguished homomorphism. Let $\pi_{1}: \mathfrak{A} \mid \mathscr{L} \rightarrow A_{z_{0}}(V)$ be the homomorphism defined by $\pi_{1}(u, v)+\mathscr{L}=v$. Then $R\left(m_{0}\right) \circ \pi_{1}$ is a homomorphism of $\mathfrak{A} \mid \mathscr{L}$ and for $f \in H^{\infty}(U)$ with $T(f)=(u, v)$

$$
\begin{aligned}
R\left(m_{0}\right) \circ \pi_{1}\left(\Theta\left(f \mid \mathscr{M}_{z_{0}}(U)\right)\right) & =R\left(m_{0}\right)(v) \\
& =R\left(m_{0}\right)\left(P_{0} f+\sum_{n=1}^{\infty} P_{n} f\left(z_{0}\right)\right) \\
& =R\left(m_{0}\right)\left(P_{0} f\right)+\sum_{n=1}^{\infty} P_{n} f\left(z_{0}\right) \\
& =\Phi\left(R\left(m_{0}\right)\right)(f)=m_{0}(f) .
\end{aligned}
$$

Hence $R\left(m_{0}\right) \circ \pi_{1}$ extends $m_{0} \circ \Theta^{-1}$ to $\mathfrak{A} \mid \mathscr{L}$.

$\Theta\left(A_{z_{0}}(U)\right)$ is a pretty large subalgebra of $\mathfrak{A} \mid \mathscr{L}$. In particular, we have 
$\Theta\left(A_{z_{0}}(U)\right) \supset H_{0}^{\infty}(\Delta \times N)+\mathscr{L}$. Indeed it is particularly easy to see that if $g \in H_{0}^{\infty}(\Delta \times N)$ then

$$
f(z)=\sum g\left(L_{n}(z), n\right)-\sum g\left(L_{n}\left(z_{0}\right), n\right)
$$

defines an element of $H^{\infty}(U)$ for which $\Theta\left(f \mid \mathscr{M}_{z_{0}}(U)\right)=g+\mathscr{L}$.

Let $m_{0} \in \mathscr{M}_{z_{0}}(U)$ be a homomorphism of $A_{z_{0}}(U)$ which is not a distinguished homomorphism. Then there is an $f \in H^{\infty}(U)$ for which

$$
m_{0}(f) \neq m_{0}\left(P_{0} f\right)+\sum_{n=1}^{\infty} P_{n} f\left(z_{0}\right) .
$$

Let $g=f-P_{0} f-\sum_{n=1}^{\infty} P_{n} f\left(z_{0}\right)$. Then by Lemma 2.3 and Lemma 2.4 and a little calculation (the "main term" $\left(P_{0} g\right)\left(\alpha_{n}\right)$ is zero here!)

$$
\Theta\left(g \mid \mathscr{M}_{z_{0}}(U)\right) \in H_{0}^{\infty}(\Delta \times N)+\mathscr{L}
$$

and $m_{0}(g) \neq 0$. This shows that the homomorphism which $m_{0}$ induces on $H_{0}^{\infty}(\Delta \times N)+\mathscr{L}$ is nonzero. This homomorphism is given by a nonzero homomorphism of $H_{0}^{\infty}(\Delta \times N)$ which vanishes on $\mathscr{L}$, which in turn is the restriction of a homomorphism of $H^{\infty}(\Delta \times N)$. Let $\psi$ be such a homomorphism of $H^{\infty}(\Delta \times N)$. Since $\psi$ is nonzero on $H_{0}^{\infty}(\Delta \times N)$ it will satisfy $\psi(Z) \neq 0$, where $Z(\lambda, n)=\lambda$. Let $\pi_{2}: \mathfrak{A} \rightarrow H^{\infty}(\Delta \times N)$ be defined by $\pi_{2}(u, v)=u$. Now $\psi \circ \pi_{2}$ vanishes on $\mathscr{L}$ and so induces a homomorphism $p$ of $\mathfrak{A} \mid \mathscr{L}$. We will show that $p$ extends $m_{0} \circ \Theta^{-1}$ to $\mathfrak{A} \mid \mathscr{L}$.

Let $f \in H^{\infty}(U)$ and let $g=f-P_{0} f-\sum_{n=1}^{\infty} P_{n} f\left(z_{0}\right)$. Then

$$
\Theta\left(g \mid \mathscr{M}_{z_{0}}(U)\right) \in H_{0}^{\infty}(\Delta \times N)+\mathscr{L}
$$

so that, by the choice of $\psi$, we will have $m_{0}(g)=p\left(\Theta\left(g \mid \mathscr{M}_{z_{0}}(U)\right)\right)$. If we could show that $m_{0}(f)=p\left(\Theta\left(f \mid \mathscr{M}_{z_{0}}(Y)\right)\right)$ for all $f \in H^{\infty}(V)$ we would be done.

In $\S 4$ we observed that the subalgebra of $H^{\infty}(\Delta \times N)$ of functions which are constant on each slice $\Delta \times\{n\}$ is isometrically isomorphic to $l^{\infty}$. Let $\omega_{0}$ be the homomorphism of $l^{\infty}$ determined by the value of $\psi$ on this algebra.

Let $F: \beta(N) \rightarrow \mathscr{M}(V)$ be the continuous extension of $F(n)=\alpha_{n}$ defined by $F(\omega)(f)=\omega\left(\left\{f\left(\alpha_{n}\right)\right\}\right)$ for all $\omega \in \beta(N)$ and $f \in H^{\infty}(V)$.

Suppose that we knew that $F\left(\omega_{0}\right)=R\left(m_{0}\right)$. For $f \in H^{\infty}(V)$ we have $\Theta\left(f \mid \mathscr{M}_{z_{0}}(U)\right)$ $=(u, v)+\mathscr{L}$ where $u(\lambda, n)=f\left(\alpha_{n}\right)$ and $v=f \mid \mathscr{M}_{z_{0}}(U)$ because all the terms in the sum defining $u(\lambda, n)$ vanish, save $\left(P_{0} f\right)\left(\alpha_{n}\right)=f\left(\alpha_{n}\right)$. Hence if $F\left(\omega_{0}\right)=R\left(m_{0}\right)$ we would have

$$
\begin{aligned}
p\left(\Theta\left(f \mid \mathscr{M}_{z_{0}}(U)\right)\right) & =p((u, v)+\mathscr{L})=\psi(u) \\
& =\omega_{0}\left(\left\{f\left(\alpha_{n}\right)\right\}\right)=F\left(\omega_{0}\right)(f) \\
& =R\left(m_{0}\right)(f)=m_{0}(f) .
\end{aligned}
$$

We will use Lemma 3.6 and Lemma 4.6 to show that $F\left(\omega_{0}\right)=R\left(m_{0}\right)$. For $P \subset N$ let $h_{P}=\sum_{n \in P} L_{n}-\sum_{n \in P} L_{n}\left(z_{0}\right)$. Let $h=h_{N}$. It is easy to see with the help of Lemmas 2.1 and 2.4 that

$$
\Theta\left(h_{P} \mid \mathscr{M}_{z_{0}}(U)\right)=Z_{P}+\mathscr{L}
$$


where $Z_{P}(\lambda, n)=\lambda$ if $n \in P$ and $Z_{P}(\lambda, n)=0$ if $n \in P^{c}$. Hence by Lemma 4.6, $\omega_{0}$ is characterized by the fact that $m_{0}\left(h_{P}\right) \neq 0$ and $f \in H^{\infty}(V)$ with $\left|f\left(\alpha_{n}\right)\right| \geqq \varepsilon$ for $n \in P$ implies that $\left|\omega_{0}\left(\left\{f\left(\alpha_{n}\right)\right\}\right)\right| \geqq \varepsilon$. Suppose that $f \in H^{\infty}(V)$ and $\left|m_{0}(f)\right|>\varepsilon$. Note that $m_{0}(h)=\psi(Z) \neq 0$. Let

$$
P=\left\{n|| f\left(\alpha_{n}\right)|\geqq \varepsilon| m_{0}(h) \mid\right\} .
$$

By Lemma 3.6 if $m_{0}\left(h_{P}\right) \neq 0$ then

$$
\left|m_{0}(f)\right| \leqq \varepsilon\left|m_{0}(h)\right| /\left|m_{0}(h)\right|=\varepsilon .
$$

Hence $m_{0}\left(h_{P^{c}}\right)=0$ and $m_{0}\left(h_{P}\right)=m_{0}\left(h_{P}\right)+m_{0}\left(h_{P^{c}}\right)=m_{0}(h)=\psi(Z) \neq 0$. Hence by Lemma 4.6 we have

$$
\left|F\left(\omega_{0}\right)(f)\right|=\left|\omega_{0}\left(\left\{f\left(\alpha_{n}\right)\right\}\right)\right| \geqq \varepsilon\left|m_{0}(h)\right| .
$$

In particular we have that $F\left(\omega_{0}\right)(f)=0$ implies that $m_{0}(f)=0$ for all $f \in H^{\infty}(V)$, i.e., $F\left(\omega_{0}\right)=R\left(m_{0}\right)$. The theorem is completely proved.

\section{The corona theorem and the parts theorem.}

THEOREM 6.1. Suppose that $V$ is a complex domain and $U$ is obtained from $V$ by deleting a hyperbolically-rare sequence of closed disks. If $V$ is dense in $\mathscr{M}(V)$ then $U$ is dense in $\mathscr{M}(U)$.

Proof. Let $m_{0} \in \mathscr{M}_{z_{0}}(U)$. If $z_{0} \in \bigcup \partial \Delta_{n}$ then that $m_{0}$ is in the closure of $U$ follows from the corona theorem for $\Delta$. Assume that $z_{0} \in \partial V$.

If $m_{0}$ is a distinguished homomorphism it is immediate that $m_{0}$ is in the closure of $U$. For if $\left\{z_{\lambda}\right\}$ is a net in $V$ which converges to $R\left(m_{0}\right)$ and $z_{\lambda}^{\prime}$ is a point in $V \backslash \bigcup D_{n}$ whose distance to $z_{\lambda}$ is not more than twice the distance of $z_{\lambda}$ to $V \backslash \cup D_{n}$, then $\left\{z_{\lambda}^{\prime}\right\}$ converges to $m_{0}$.

Now assume that $m_{0}$ is not distinguished. As in the proof of Theorem 5.2, let $p$ be an extension of $m_{0} \circ \Theta^{-1}$ from $\Theta\left(A_{z_{0}}(U)\right)$ to $\mathfrak{X} \mid \mathscr{L}$ and let $\psi$ be a homomorphism of $H^{\infty}(\Delta \times N)$ for which $p((u, v)+\mathscr{L})=\psi(u)$ for all $(u, v) \in \mathfrak{A}$. Let $f_{1}, \ldots, f_{n} \in H^{\infty}(U)$ and $\varepsilon>0$ be given. Let $\Theta\left(f_{i} \mid \mathscr{M}_{z_{0}}(U)\right)=\left(u_{i}, v_{i}\right)+\mathscr{L}$. For $q \in N$ let $I_{q} \in H^{\infty}(\Delta \times N)$ be the function defined by $I_{q}(z, n)=1$ if $n \geqq q$ and $I_{q}(z, n)=0$ if $n<q$. Note that $\psi\left(I_{q}\right)=1$. Also remember that $Z \in H^{\infty}(\Delta \times N)$ was defined by $Z(\lambda, n)=\lambda$ and we have that $\psi(Z) \neq 0$. By the corona theorem for $H^{\infty}(\Delta \times N)$ there exists a $(\lambda, k) \in \Delta \times N$ for which

$$
\begin{gathered}
\left|u_{i}(\lambda, k)-\psi\left(u_{i}\right)\right|<\varepsilon \text { for } i=1, \ldots, n, \\
\left|I_{q}(\lambda, k)-\psi\left(I_{q}\right)\right|<\varepsilon, \quad|Z(\lambda, k)-\psi(Z)|<\frac{|\psi(Z)|}{2} .
\end{gathered}
$$

In particular we have that $k>q$ and $|\lambda| \geqq|\psi(Z)| / 2$. If $q$ was chosen sufficiently large the last two inequalities guarantee that $L_{k}^{-1}(\lambda) \in D_{k} \mid \Delta_{k}$. Hence if $q$ is larger than the $N_{0}$ of Lemma 2.4, then $L_{k}^{-1}(\lambda) \in U$ and, since $m_{0}\left(f_{i}\right)=\psi\left(u_{i}\right)$,

$$
\begin{aligned}
\left|f_{i}\left(L_{k}^{-1}(\lambda)\right)-m_{0}\left(f_{i}\right)\right| \leqq & \left|f_{i}\left(L_{k}^{-1}(\lambda)\right)-u_{i}(\lambda, k)\right| \\
& +\left|u_{i}(\lambda, k)-\psi\left(u_{i}\right)\right|<2 \varepsilon \quad \text { for } i=1, \ldots, n .
\end{aligned}
$$


With the aid of the representation of $A_{z_{0}}(U)$ as a subalgebra of $\mathfrak{A} \mid \mathscr{L}$ we can describe the fiber $\mathscr{M}_{z_{0}}(U)$ in some detail and, in particular, can characterize the Gleason parts of $\mathscr{M}_{z_{0}}(U)$. To begin with, each homomorphism of $\mathfrak{A}$ is of the form $(u, v) \rightarrow \psi(u)$ where $\psi \in \mathscr{M}\left(H^{\infty}(\Delta \times N)\right)$ or $(u, v) \rightarrow \varphi(v)$ where $\varphi \in \mathscr{M}_{z_{0}}(V)$ so that we can identify the maximal ideal space of $\mathfrak{A}$ with the disjoint union of $\mathscr{M}\left(H^{\infty}(\Delta \times N)\right)$ and $\mathscr{M}_{z_{0}}(V)$. The maximal ideal space of $\mathfrak{A} \mid \mathscr{L}$ can then be identified with the set of homomorphisms of $\mathfrak{A}$ which vanish on $\mathscr{L}$ and hence with the disjoint union of $\mathscr{M}_{z_{0}}(V)$ and a subset $X$ of $\mathscr{M}\left(H^{\infty}(\Delta \times N)\right)$.

For $A \subset \beta(N)$ let $\mathscr{D}(A)$ be as defined in $\S 4$. Let $F: \beta(N) \rightarrow \mathscr{M}(V)$ be the continuous extension of $F(n)=\alpha_{n}$. It is easy to see that $X=\mathscr{D}\left(F^{-1}\left(\mathscr{M}_{z_{0}}(V)\right)\right)$.

Since each homomorphism of $\Theta\left(A_{z_{0}}(U)\right)$ extends to $\mathfrak{X} \mid \mathscr{L}$ we can identify $\mathscr{M}_{z_{0}}(U)$ with the space $Y$ obtained from $\mathscr{M}_{z_{0}}(V) \cup X$ by identifying points which are not separated by $\Theta\left(A_{z_{0}}(U)\right)$.

THEOREM 6.2. $\mathscr{M}_{z_{0}}(U)$ is homeomorphic to the space $Y$ obtained from $\mathscr{M}_{z_{0}}(V) \cup \mathscr{D}\left(F^{-1}\left(\mathscr{M}_{z_{0}}(V)\right)\right)$ by identifying along $F$, i.e. by identifying for each $\psi \in \mathscr{M}_{z_{0}}(V)$, the set $\{\psi\} \cup\left(\{0\} \times F^{-1}(\psi)\right)$ to a point. Each element of $A_{z_{0}}(U)$ will be analytic on each analytic subset of $Y$.

Proof. $\Theta\left(A_{z_{0}}(U)\right)$ contains $H_{0}^{\infty}(\Delta \times N)+\mathscr{L}$ and $H_{0}^{\infty}(\Delta \times N)+\mathscr{L}$ separates the points of $X \backslash\left(\{0\} \times F^{-1}\left(\mathscr{M}_{z_{0}}(V)\right)\right)$. Also $Z+\mathscr{L}$ is nonzero at each point of $X \backslash\left(\{0\} \times F^{-1}\left(\mathscr{M}_{z_{0}}(V)\right)\right)$ and is zero on $\mathscr{M}_{z_{0}}(V)$, so that each point of $\mathscr{M}_{z_{0}}(V)$ is separated from each point of $X \backslash\left(\{0\} \times F^{-1}\left(\mathscr{M}_{z_{0}}(V)\right)\right)$. $\Theta\left(A_{z_{0}}(U)\right)$ contains $\Theta\left(A_{z_{0}}(V)\right)$ and $\Theta\left(A_{z_{0}}(V)\right)$ separates the points of $\mathscr{M}_{z_{0}}(V)$. If $F(\omega) \in \mathscr{M}_{z_{0}}(V)$ then for $f \in H^{\infty}(U)$ with $T(f)=(u, v)$ we have (since $L_{n}^{-1}(0)=\infty$ and $\left(P_{n} f\right)(\infty)=0$ ),

$$
\begin{aligned}
(0, \omega)(u) & =\omega\left(\left\{P_{0} f\left(\alpha_{n}\right)+\sum_{m \geqq 1 ; m \neq n} P_{m} f\left(\alpha_{n}\right)\right\}\right) \\
& =\omega\left(\left\{P_{0} f\left(\alpha_{n}\right)\right\}\right)+\sum_{m=1}^{\infty} P_{m} f\left(z_{0}\right) \quad\left(\text { Lemma } 2.1 \text { and the fact } \alpha_{n} \rightarrow z_{0}\right) \\
& =F(\omega)\left(P_{0} f\right)+\sum_{m=1}^{\infty} P_{m} f\left(z_{0}\right)=F(\omega)(v) .
\end{aligned}
$$

This calculation shows that for each $\psi \in \mathscr{M}_{z_{0}}(V)$ the points of the set

$$
\{\psi\} \cup\left(\{0\} \times F^{-1}(\psi)\right)
$$

cannot be separated and that $\left(0, \omega_{1}\right)$ and $\left(0, \omega_{2}\right)$ can be separated if $F\left(\omega_{1}\right) \neq F\left(\omega_{2}\right)$. That does it.

Corollary 6.3. Let $\psi \in \mathscr{M}_{z_{0}}(V)$. If $\psi$ is not in the closure of $\left\{\alpha_{n}\right\}$ in $\mathscr{M}(V)$ then $\mathscr{M}_{\psi}(U)=\{\Phi(m)\}$, i.e. $\psi$ has a unique extension to $H^{\infty}(U)$ [Theorem 3.4]. If $\psi$ is in the closure of $\left\{\alpha_{n}\right\}$ in $\mathscr{M}(V)$ then $\mathscr{M}_{\psi}(U)$ is homeomorphic to $\mathscr{D}\left(F^{-1}(\psi)\right)$ with $0 \times F^{-1}(\psi)$ identified to $\Phi(\psi)$. 
Let $P$ be a Gleason part of $H^{\infty}(U)$ and suppose that $\Phi(\psi) \in P$. Clearly $P$, when considered as a subset of $Y$, contains $\Delta \times F^{-1}(\psi)$ (with $0 \times F^{-1}(\psi)$ identified to $\Phi(\psi))$. In fact, as the function $Z+\mathscr{L}$ with the aid of Theorem 4.5 shows, we will have $P \cap \mathscr{M}_{\psi}(U)=\Delta \times F^{-1}(\psi)$ (with $0 \times F^{-1}(\psi)$ identified to $\Phi(\psi)$ ).

If $P \cap \mathscr{M}_{\psi}(U) \neq \varnothing$ but $\Phi(\psi) \notin P$ we will have

$$
P \cap \mathscr{M}_{\psi}(U) \subset \mathscr{D}\left(F^{-1}(\psi)\right) \backslash \Delta \times F^{-1}(\psi) .
$$

The functions $Z_{P}+\mathscr{L}$ show that if a part intersects $\mathscr{D}\left(F^{-1}(\psi)\right) \mid\left(\Delta \times F^{-1}(\psi)\right)$ it must be contained entirely in this set. Corollary 4.4 shows that each part in this set is either a single point or an analytic disk. Hence we have

THEOREM 6.4. Let $P$ be a Gleason part of $H^{\infty}(U)$. Let $\psi \in \mathscr{M}(V)$. If $\Phi(\psi) \in P$ then $P \cap \mathscr{M}_{\psi}(U)$ is homeomorphic to $\Delta \times F^{-1}(\psi)$ with $0 \times F^{-1}(\psi)$ identified to $\Phi(\psi)$. Each $f \in H^{\infty}(U)$ will be analytic on each "slice" of $\Delta \times F^{-1}(\psi)$. If $P \cap \mathscr{M}_{\psi}(U) \neq \varnothing$ but $\Phi(\psi) \notin P$ then $P \subset \mathscr{M}_{\psi}(U)$ and $P$ is either a single point or an analytic disk.

Our final statement of the parts theorem is the following:

THEOREM 6.5. Let $P$ be a Gleason part of $H^{\infty}(U)$. If $P$ contains no distinguished homomorphism, then $P$ is either a single point or an analytic disk. Let $P_{0}$ be the Gleason part that contains $U$. If $P \neq P_{0}$ contains a distinguished homomorphism then $R(P)$ is a Gleason part of $H^{\infty}(V)$ and $P$ is homeomorphic to the space obtained from $R(P) \cup \Delta \times F^{-1}(R(P))$ by identifying, for each $\psi \in R(P)$, the set $\{0\} \times F^{-1}(\psi)$ to the point $\psi$. Each $f \in H^{\infty}(U)$ will be analytic on each set $\Delta \times\{\omega\}$ with $\omega \in F^{-1}(R(P))$. For each $z_{0} \in \partial V, P_{0} \cap \mathscr{M}_{z_{0}}(U)$ is a Gleason part of $A_{z_{0}}(U)$ and

$$
R\left(P_{0} \cap \mathscr{M}_{z_{0}}(U)\right)=R\left(P_{0}\right) \cap \mathscr{M}_{z_{0}}(V) .
$$

$P_{0} \cap \mathscr{M}_{z_{0}}(U)$ will be homeomorphic to

$$
\left(R\left(P_{0}\right) \cap \mathscr{M}_{z_{0}}(V)\right) \cup \Delta \times F^{-1}\left(R\left(P_{0}\right) \cap \mathscr{M}_{z_{0}}(V)\right)
$$

with, for each $\psi \in R\left(P_{0}\right) \cap \mathscr{M}_{z_{0}}(V)$, the set $\{0\} \times F^{-1}(\psi)$ identified to the point $\psi$.

7. The unit disk. When $V$ is the unit disk $\Delta$, the Gleason parts of $U$ depend only on the nature of the sequence $\left\{\alpha_{n}\right\}$ relative to the hyperbolic geometry of $\Delta$. This is a consequence of Theorem 6.5 and Hoffman's [7] characterization of the Gleason parts of $H^{\infty}(\Delta)$. We will consider several examples where the geometric nature of $\left\{\alpha_{n}\right\}$ is easy to describe.

Let $\chi$ be the hyperbolic metric in $\Delta$.

Define a "flower" to be an analytic set of the form $\Delta \times K$ with $\{0\} \times K$ identified to a point, where $K$ is a closed subset of $\beta(N) \mid N$. For each $\lambda \in \Delta$ let $K_{\lambda}$ be a closed subset of $\beta(N) \mid N$. Define a "garden" to be an analytic set of the form $\Delta \cup \bigcup\left\{\Delta \times K_{\lambda}: \lambda \in \Delta\right\}$ with each $\{0\} \times K_{\lambda}$ identified to $\lambda$. Call a garden an " $A$ garden" if $K_{\lambda}=\varnothing$ for $\lambda \notin A$ and $K_{\lambda}=\{\lambda\}$ for $\lambda \in A_{n}$. Here $A$ is a subset of $\Delta$. Call an $A$-garden an "interpolating garden" if $A$ is an interpolating sequence. Call a garden a "congested garden" if each $K_{\lambda}$ has cardinality $2^{c}$. 
THEOREM 7.1. Let $U=\Delta \backslash \bigcup \Delta_{n}$ with $\Delta_{n}$ hyperbolically-rare in $\Delta$. Let $\alpha_{n}$ $=$ center $\Delta_{n}$.

(1) Each Gleason part of $H^{\infty}(U)$ is either a point, a flower, or a garden.

(2) If $\left\{\alpha_{n}\right\}$ is interpolating, then each Gleason part of $H^{\infty}(U)$ is either a point or an interpolating garden.

(3) If

$$
\lim _{N \rightarrow \infty} \inf _{n, m \geqq N} \chi\left(\alpha_{n}, \alpha_{m}\right)=\infty
$$

then each Gleason part of $H^{\infty}(U)$ is a point or a flower.

(4) If $\left\{\alpha_{n}\right\}$ is interpolating and

$$
\lim _{N \rightarrow \infty} \inf _{n, m \geqq N} \chi\left(\alpha_{n}, \alpha_{m}\right)=\infty,
$$

then each Gleason part of $H^{\infty}(U)$ is a point, an analytic disk, or two analytic disks with the origins identified.

(5) If $\left\{\alpha_{n}\right\}$ is invariant under a discontinuous group of conformal maps of $\Delta$ which act transitively on $\left\{\alpha_{n}\right\}$, then each Gleason part of $H^{\infty}(U)$ is a point, a flower, or an $\left\{\alpha_{n}\right\}$-garden.

(6) If $\left\{\alpha_{n}\right\}$ is hyperbolically dense as $|z| \rightarrow 1$, i.e. if

$$
\lim _{\delta \rightarrow 1} \sup _{|z| \geq \delta} \inf _{n} \chi\left(z, \alpha_{n}\right)=0,
$$

then each Gleason part of $H^{\infty}(U)$ is a point, a flower, or a congested garden.

Proof. (1) Since each part of $H^{\infty}(\Delta)$ is a point or an analytic disk, (1) is a restatement of Theorem 6.5.

(2) An interpolating sequence intersects no point part of $H^{\infty}(U)$ and intersects an analytic disk of $H^{\infty}(U)$ in an interpolating sequence.

(3) Such a sequence intersects a nontrivial part of $H^{\infty}(U)$ in at most one point.

(4) Follows from (2) and (3).

(5) Such a sequence intersects a nontrivial part of $H^{\infty}(U)$ in a set conformally equivalent to $\left\{\alpha_{n}\right\}$.

(6) Such a sequence intersects every point of $\mathscr{M}\left(H^{\infty}(\Delta)\right) \backslash \Delta$.

8. General domains. In the announcement [1], we considered domains obtained by removing from the punctured disk $\Delta^{\prime}=\Delta \mid\{0\}$ a hyperbolically-rare sequence of disks $\left\{\Delta_{n}\right\}$ which accumulate at 0 . Our methods are greatly simplified in this case.

We will now consider a domain obtained from $\Delta^{\prime}$ by deleting a sequence of closed sets $\left\{E_{n}\right\}$ for which $E_{n} \subset \Delta_{n}$ with $\left\{\Delta_{n}\right\}$ a hyperbolically-rare sequence of closed disks which accumulate at 0 . In this case we will also call the $E_{n}$ hyperbolically-rare.

Let $D=\Delta^{\prime} \backslash \bigcup E_{n}$. If $f \in H^{\infty}(D)$ then

$$
\frac{1}{2 \pi i} \int_{\partial \Delta_{n}} \frac{f(\xi) d \xi}{z-\xi}
$$

is an analytic function on $\Delta_{n}^{c}$ which extends to a bounded analytic function on $E_{n}^{c}$. 
If we denote this function by $P_{n} f$ the resulting mappings $P_{n}$ from $H^{\infty}(D)$ into $H^{\infty}\left(E_{n}^{c}\right)$ are uniformly bounded. Let $H^{\infty}\left(\left\{E_{n}^{c}\right\}\right)$ be the uniform algebra of all $\left\{f_{n}\right\}$ with $f_{n} \in H^{\infty}\left(E_{n}^{c}\right)$ and $\left\|\left\{f_{n}\right\}\right\|=\sup \left\|f_{n}\right\|<\infty$. Let $H_{0}^{\infty}\left(\left\{E_{n}^{c}\right\}\right)$ be the algebra of all $\left\{f_{n}\right\} \in H^{\infty}\left(\left\{E_{n}^{c}\right\}\right)$ with $f_{n}(\infty)=0$ for all $n$. Let $\mathscr{M}\left(H^{\infty}\left(\left\{E_{n}^{c}\right\}\right)\right)$ be the maximum ideal space of $H^{\infty}\left(\left\{E_{n}^{c}\right\}\right)$ and $\mathscr{M}\left(H_{0}^{\infty}\left(\left\{E_{n}^{c}\right\}\right)\right)$ be the maximal ideal space of the Banach algebra obtained by adjoining the constants to $H_{0}^{\infty}\left(\left\{E_{n}^{c}\right\}\right)$. Now $H^{\infty}\left(\left\{E_{n}^{c}\right\}\right)$ can be identified with the Banach space direct sum of $H_{0}^{\infty}\left(\left\{E_{n}^{c}\right\}\right)$ and $l^{\infty}$. For each $\psi \in \mathscr{M}\left(H_{0}^{\infty}\left(\left\{E_{n}^{c}\right\}\right)\right)$ let $\omega$ be the ultrafilter of all $P \subset N$ for which $\psi\left(\left\{f_{n}\right\}\right)=0$ for all $\left\{f_{n}\right\} \in H_{0}^{\infty}\left(\left\{E_{n}^{c}\right\}\right)$ with $f_{n} \equiv 0$ for $n \in P$. Then $\omega$ defines a homomorphism of $l^{\infty}$. It is easy to see that the linear functional on $H^{\infty}\left(\left\{E_{n}^{c}\right\}\right)$ defined by $\psi$ and $w$ is a homomorphism and that this homomorphism is the unique extension of $\psi$ to $H^{\infty}\left(\left\{E_{n}^{c}\right\}\right)$. This shows that $\mathscr{M}\left(H_{0}^{\infty}\left(\left\{E_{n}^{c}\right\}\right)\right)$ is obtained from $\mathscr{M}\left(H^{\infty}\left(\left\{E_{n}^{c}\right\}\right)\right)$ by identifying the set of homomorphisms which vanish on $H_{0}^{\infty}\left(\left\{E_{n}^{c}\right\}\right)$ to a point. Now the $E_{n}^{c}$ can be identified with a subset of $\mathscr{M}\left(H^{\infty}\left(E_{n}^{c}\right)\right)$ by identifying each point $\lambda \in E_{n}^{c}$ with the homomorphism $\left\{f_{n}\right\} \rightarrow f_{n}(\lambda)$. Denote this homomorphism by $(\lambda, n)$. The closure of $E_{n}^{c} \times\{n\}$ in $\mathscr{M}\left(H^{\infty}\left(\left\{E_{n}^{c}\right\}\right)\right)$ is an open-closed subset of $\mathscr{M}\left(H^{\infty}\left(\left\{E_{n}^{c}\right\}\right)\right)$. Let $X$ be the set obtained from

$$
\mathscr{M}\left(H^{\infty}\left(\left\{E_{n}^{c}\right\}\right)\right) \bigcup_{n} \mathrm{Cl}\left(E_{n}^{c} \times\{n\}\right)
$$

by identifying the set of homomorphisms which vanish on $H_{0}^{\infty}\left(\left\{E_{n}^{c}\right\}\right)$ to a point and let $A$ be the restriction of $H_{0}^{\infty}\left(\left\{E_{n}^{c}\right\}\right)$ to $X$ with the constant adjoined. Our methods now give, exactly as in [1], that the fiber algebra at 0 in $H^{\infty}(D)$ is isometrically (algebra) isomorphic to $A$.

In order to use this to prove a corona theorem we need the following definition:

For each complex domain $D, \delta>0$ and integer $n$, let $C(\delta, n, D)$ be the smallest constant for which given $f_{1}, \ldots, f_{n} \in H^{\infty}(D)$ with $\left\|f_{i}\right\| \leqq 1$ and $\sum\left|f_{i}\right| \geqq \delta$ there are $g_{1}, \ldots, g_{n} \in H^{\infty}(D)$ with $\left\|g_{i}\right\| \leqq C(\delta, n, D)$ and $\sum f_{i} g_{i}=1$. If no such constant exists let $C(\delta, n, D)=\infty$.

Note that the constants $C(\delta, n, D)$ are preserved under conformal equivalence. We have

THEOREM 8.1. Let $E_{n}$ be a hyperbolically-rare sequence of closed sets contained in $\Delta$ which accumulate at 0 and let $D=\Delta^{\prime} \backslash \cup E_{n}$. Then $D$ is dense in $\mathscr{M}(D)$ if and only if each $E_{n}^{c}$ is dense in $\mathscr{M}\left(E_{n}^{c}\right)$ and for each $\delta, n$ there is an integer $N_{0}$ such that

$$
\sup _{m \geqq N_{0}} C\left(\delta, n, E_{m}^{c}\right)<\infty
$$

This theorem follows easily from the fact that the given condition is equivalent to the density of $\bigcup_{n} E_{n}^{c} \times\{n\}$ in $\mathscr{M}\left(H^{\infty}\left(\left\{E_{n}^{c}\right\}\right)\right)$.

Any complex domain $D_{n}$ can be written as a union of an increasing sequence $\left\{D_{n}\right\}$ of finitely connected domains. It follows by a simple normal families argument, that if $\sup _{m} C\left(\delta, n, D_{m}\right)<\infty$ for each $\delta, n$, then $D$ is dense in $\mathscr{M}(D)$. Now each finitely connected domain is conformally equivalent to a domain bounded by a 
finite number of disjoint circles. Hence we have that if the corona theorem fails for some complex domain then there is a sequence of domains $D_{n}$, each of which has as boundary the disjoint union of a finite number of circles, such that for some $\delta, n, \sup _{m} C\left(\delta, n, D_{m}\right)=\infty$.

Combining this with Theorem 8.1 gives

COROLlARY 8.2. If the corona theorem fails for some complex domain, then it fails for a domain obtained from the open unit disk by removing a sequence of closed disks which accumulate only at 0.

When $D$ is obtained from $\Delta^{\prime}$ by removing a hyperbolically-rare sequence of closed disks which converge to 0 , the Gleason parts of $H^{\infty}(D)$ are particularly easy to describe. In particular [1], the Gleason part of $H^{\infty}(D)$ containing $D$, denoted by $P_{0}(D)$, is equal to the union of $D$ and a set homeomorphic to $\Delta \times \hat{\beta}(N)$ with $\{0\} \times \hat{\beta}(N)$ identified to the point $\varphi_{0}$, where $\beta(N)$ is the Stone-Čech compactification of the integers $N, \hat{\beta}(N)=\beta(N) \backslash N$, and $\varphi_{0}$ is defined by

$$
\varphi_{0}(f)=\frac{1}{2 \pi i} \int_{\partial D} \frac{f(\xi) d \xi}{\xi} .
$$

We have the following similar result for more general domains.

THEOREM 8.3. Let $D$ be obtained by removing from $\Delta^{\prime}$ the hyperbolically-rare sequence of closed sets $E_{n}$ which accumulate at 0 . Let $\Delta_{n}$ be a hyperbolically-rare sequence of closed disks for which $E_{n} \subset \Delta_{n}$. Let $\gamma\left(E_{n}\right)$ be the analytic capacity of $E_{n}$. If

$$
\lim \sup \left(\gamma\left(E_{n}\right) / \operatorname{rad} \Delta_{n}\right)>0,
$$

then the Gleason part of $H^{\infty}(D)$ containing $D$ contains in the fiber at 0 a set homeomorphic to $\Delta \times \hat{\beta}(N)$ with $\{0\} \times \hat{\beta}(N)$ identified to the homomorphism $\varphi_{0}$ defined by

$$
\varphi_{0}(f)=\frac{1}{2 \pi i} \int_{\cup \partial \Delta_{n}} \frac{f(\xi) d \xi}{\xi} .
$$

Proof. Let

$$
P=\left\{n \in N \mid \frac{\gamma\left(E_{n}\right)}{\operatorname{rad} \Delta_{n}}>\frac{1}{2} \lim \sup \frac{\gamma\left(E_{n}\right)}{\operatorname{rad} \Delta_{n}}\right\}
$$

There will be an $R>0$, and $r>0$, and for each $n \in P$ a function $f_{n} \in H^{\infty}\left(E_{n}^{c}\right)$ such that $\left\|f_{n}\right\| \leqq 1, f_{n}(\infty)=0$, and $f_{n}$ is one-to-one on $\left\{z|| z-\operatorname{center} \Delta_{n} \mid \geqq R \operatorname{rad} \Delta_{n}\right\}$, mapping that set one-to-one including $\Delta_{r}=\{z|| z \mid \leqq r\}$. For each $\lambda \in \Delta_{r}$ and $n \in P$ let $G(\lambda, n)$ be the homomorphism $\left\{g_{m}\right\} \rightarrow g_{n}(z)$ where $z$ is the unique point in $\left\{z|| z-\right.$ center $\left.\Delta_{n} \mid \geqq R \operatorname{rad} \Delta_{n}\right\}$ for which $f_{n}(z)=\lambda$. The resulting mapping $G: \Delta_{r} \times P \rightarrow \mathscr{M}\left(H^{\infty}\left(\left\{E_{n}^{c}\right\}\right)\right)$ is continuous and hence extends continuously to $\Delta_{r} \times \beta(P)$. If $\lambda_{1} \neq \lambda_{2}$ then for each $n \in P$

$$
\left\{f_{m}\right\}\left(G\left(\lambda_{1}, n\right)\right)-\left\{f_{m}\right\}\left(G\left(\lambda_{2}, n\right)\right)=\lambda_{1}-\lambda_{2}
$$

and hence for all $\omega \in \beta(P)$

$$
\left\{f_{m}\right\}\left(G\left(\lambda_{1}, \omega\right)\right)-\left\{f_{m}\right\}\left(G\left(\lambda_{2}, \omega\right)\right)=\lambda_{1}-\lambda_{2}
$$


Also $G\left(\lambda_{1}, \omega_{1}\right) \neq G\left(\lambda_{2}, \omega_{2}\right)$ if $\omega_{1} \neq \omega_{2}$ since $H^{\infty}\left(\left\{E_{n}^{c}\right\}\right)$ contains $l^{\infty}$. Hence $G$ is oneto-one on $\Delta_{r} \times \beta(P)$ and is hence a homeomorphism. The theorem now follows from the representation theorem.

THEOREM 8.4. Let $U$ be a planar domain which contains 0 and for which each $C(\delta, n, U)$ is finite. Let $D$ be a domain obtained by removing from $\Delta^{\prime}$ a hyperbolicallyrare sequence of closed sets $E_{n}$ which accumulate at 0 and for which each $E_{n}^{c}$ is conformally equivalent to $U$. Then $D$ is dense in $\mathscr{M}(D)$. The Gleason part $P_{0}(D)$ of $H^{\infty}(D)$ which contains $D$ contains in the fiber $\mathscr{M}_{0}(D)$ a set which is homeomorphic to $U \times \hat{\beta}(N)$ with $\{0\} \times \hat{\beta}(N)$ identified to the distinguished homomorphism $\Phi_{0}$. Each function in $H^{\infty}(D)$ will be analytic on each slice of $U \times \hat{\beta}(N)$. If $U$ is a Gleason part of $H^{\infty}(U)$ then $P_{0}(D) \cap \mathscr{M}_{0}(D)$ is homeomorphic to $U \times \hat{\beta}(N)$ with $\{0\} \times \hat{\beta}(N)$ identified to $\Phi_{0}$ with each function in $H^{\infty}(D)$ analytic on each slice of $U \times \hat{\beta}(N)$.

Proof. $D$ is dense in $\mathscr{M}(D)$ by Theorem 8.1. The algebras $H^{\infty}\left(\left\{E_{n}^{c}\right\}\right)$ and $H^{\infty}(U \times N)$ are isometrically (algebra) isomorphic so that we may use the latter algebra in the representation of the fiber algebra. Let $T$ be the mapping of $\mathscr{M}\left(H^{\infty}(U \times N)\right)$ into $\mathscr{M}(U)$ obtained by restricting each homomorphism of $H^{\infty}(U \times N)$ to the subalgebra of all functions which are constant on each set of the form $\{z\} \times N$. Let $S$ be the mapping of $\mathscr{M}\left(H^{\infty}(U \times N)\right)$ into $\beta(N)$ obtained by restricting each homomorphism of $H^{\infty}(U \times N)$ to the subalgebra of functions which are constant on each set of the form $U \times\{n\}$. Let $G$ be the continuous mapping of $\mathscr{M}\left(H^{\infty}(U \times N)\right)$ into $\mathscr{M}(U) \times \beta(N)$ defined by $G(\psi)=(T(\psi), S(\psi))$.

Suppose that $\psi \in \mathscr{M}\left(H^{\infty}(U \times N)\right)$ and $T(\psi) \in U$. Let $\left(z_{\lambda}, n_{\lambda}\right)$ be a net in $U \times N$ which converges to $\psi$. Then $z_{\lambda}$ converges to $T(\psi)$ and $n_{\lambda}$ converges to $S(\psi)$. Using Schwarz's lemma it is immediate that $\left(T(\psi), n_{\lambda}\right)$ will converge to $\psi$. It follows that $\psi$ is given by the mapping $\left\{f_{n}\right\} \rightarrow S(\psi)\left(\left\{f_{n}(T(\psi))\right\}\right)$. Let $H$ be the mapping of $U \times \beta(N)$ into $\mathscr{M}\left(H^{\infty}(U \times N)\right)$ given by $H(z, \omega)\left(\left\{f_{n}\right\}\right)=\omega\left(\left\{f_{n}(z)\right\}\right)$. We have shown that for $\psi \in H(U \times \beta(N)), G^{-1}(G(\psi))=\{\psi\}$ and that $G \circ H$ is the identity on $U \times \beta(N)$. It follows by the elementary topology that $H$ is a homeomorphism. The theorem now follows by the representation theorem.

\section{BIBLIOGRAPHY}

1. M. Behrens, The corona conjecture for a class of infinitely connected domains, Bull. Amer. Math. Soc. 76 (1970), 387-391.

2. L. Carleson, Interpolations by bounded analytic functions and the corona problem, Ann. of Math. (2) 76 (1962), 547-559. MR 25 \#5186.

3. T. Gamelin, Uniform algebras, Prentice-Hall, Englewood Cliffs, N. J., 1969.

4. - Localization of the corona problem (to appear).

5. K. Hoffman, Analytic functions and logmodular Banach algebras, Acta Math. 108 (1962), 271-317. MR 26 \#6820.

6. - Banach spaces of analytic functions, Prentice-Hall Series in Modern Analysis, Prentice-Hall, Englewood Cliffs, N. J., 1962. MR 24 \#A2844.

7. — Bounded analytic functions and Gleason parts, Ann. of Math. (2) 86 (1967), 74-111. MR 35 \#5945. 
8. I. J. Schark, Maximal ideals in an algebra of bounded analytic functions, J. Math. Mech. 10 (1961), 735-746. MR 23 \#A2744.

9. E. L. Stout, Two theorems concerning functions holomorphic on multiply connected domains, Bull. Amer. Math. Soc. 69 (1963), 527-530. MR 27 \#275.

10. L. Zalcman, Bounded analytic functions on domains of infinite connectivity, Trans. Amer. Math. Soc. 144 (1969), 241-270.

Massachusetts Institute of Technology, Cambridge, Massachusetts 02139 